\title{
Feasibility of the Laparoscopic Total Colectomy with Ileal j-Pouch-Rectal Anastomosis in the Treatment of Chronic Constipation Refractory to Medical Therapy: Observations about a Case Report
}

\author{
Alfredo Allaria, Vincenzo Napolitano, Maria Chiara Bondanese*, Francesco Moccia, \\ Marco Cimmino, Beniamino Pascotto, Francesco Orlando, Landino Fei \\ Unit of Gastrointestinal Surgery, Department of Anaesthesiological, Surgical and Emergency Sciences \\ Second University of Naples, School of Medicine Via Pansini, Naples, Italy \\ Email: "mariachiarabondanese@libero.it
}

Received May 27, 2013; revised June 28, 2013; accepted July 6, 2013

Copyright (c) 2013 Alfredo Allaria et al. This is an open access article distributed under the Creative Commons Attribution License, which permits unrestricted use, distribution, and reproduction in any medium, provided the original work is properly cited.

\begin{abstract}
In October 2012, a 29-year-old woman affected by chronic constipation underwent surgery for a laparoscopic total colectomy with ileal j-pouch-rectal anastomosis. After a precise instrumental work-up, the patient was selected as a potential candidate for the surgical treatment after failing to respond to all other medical treatment. During the first three months following the surgery, the patient recorded 3 - 4 daily evacuations of soft stool. After the third month, the number of evacuations stabilised at 2 - 3 a day, with formed stool, and no mention of incontinence or abdominal pain. Although the laparoscopic total colectomy with ileorectal anastomosis (TC with IRA) and the laparoscopically assisted subtotal colectomy with cecorectal anastomosis (SC with CRA) are the most frequently practiced procedures in the surgical treatment of chronic constipation, the laparoscopic colectomy with ileal j-pouch has shown in our experience to be a feasible and effective procedure, with similar results to the two aforementioned procedures in terms of morbidity, mortality and quality of life. In fact, like the first two, the total colectomy with ileal j-pouch has demonstrated the advantages of laparoscopic surgery: low invasiveness, less post-operative pain or ileus, respect of parietal integrity, reduced hospitalization and, lastly, a better cosmesis.
\end{abstract}

Keywords: Total Colectomy; Chronic Constipation; Laparoscopy; Ileal j-Pouch

\section{Introduction}

The numerous advantages, by now well-recognised, of laparoscopic surgery and laparoscopically assisted surgery have created the diffusion of laparoscopic colectomy surgery for the treatment of a benign pathology like chronic constipation refractory to pharmacologic therapy. The patients selected for this type of treatment are generally young individuals with a long life expectancy and the most common procedures used are the total laparoscopic colectomy with ileorectal anastomosis (TC with IRA) and the laparoscopically assisted subtotal colectomy with cecorectal anastomosis (SC with CRA) [1-3]. We report a case of a 29-year-old woman treated with a laparoscopic total colectomy and ileal $\mathrm{j}$-pouch to assess the advantages of this type of surgery in terms of mor-

*Corresponding author. bidity, mortality and, above all, quality of life, in respect to two other patients who were treated with an ileorectal anastomosis without ileal pouch.

\section{Case Report}

A 29-year-old woman (D. R.) came to our observation in April 2012. Among her symptoms was chronic constipation, with a single evacuation of solid stool every 8 - 10 days, chronic abdominal pain, nausea and flatulence. These symptoms persisted despite pharmacologic therapy based on oral fibers and fermented milks taken daily and a diet consisting of fruit, vegetables, pulses and two litres of water a day. Evacuations only occurred after assuming osmotic laxatives, which the woman took every $8-10$ days when the suffering became intolerable. The diagnostic process which the patient underwent in our De- 
partment included: 1) a radiologic barium enema that showed a normal colon patency of the baritated column and dolichocolon with ptosis of the transverse colon; 2) the pancoloscopy, which demonsrated the absence of neoplastic lesions and/or benignant strictures; 3) the anorectal manometry, that reported the following values: anal sphincter mean basal pressure $50 \mathrm{mmHg}$, voluntary maximum contraction $110 \mathrm{mmHg}$, duration of the voluntary maximum contraction 20 seconds, rectal-anal inhibitory reflex (R.A.I.R) present, rectal sensitivity test: first threshold at $30 \mathrm{cc}$, second threshold at $70 \mathrm{cc}$, third threshold at $130 \mathrm{cc}$; 4) study of intestinal transit time through the ingestion of 50 radiographic markers (10 per day) for five days and the carrying out of an X-ray on the seventh day. The study highlighted a slow transit with the presence of 12 markers in the ascending colon, 22 in the transverse colon, 8 between the splenic flexure and the descending colon and 3 in the sigmoid colon. Therefore, all these tests had negative results except for the last one which revealed a very slow colonic transit time.

In October 2012, the patient underwent a laparoscopic total colectomy with ileal j-pouch and ileorectal anastomosis.

\section{Operative Procedure}

The surgery was carried out through the insertion of 5 trocars (3 measuring $10-12 \mathrm{~mm}$ and 2 measuring $5 \mathrm{~mm}$ ) in the following positions: above the groin, umbilicus and paramedian right and left at approximately the level of the transverse umbilical line. The rectum is then prepared and, after having sunken it with the use of an "endo-GIA" stapler, the entire colon is prepared using the "Ligasure Atlas" to section the vessels, which is carried out behind the colic margin. Once the colon is prepared, a "routine mini-laparotomy" above the groin (joining the fixture points of the trocars positioned in the umbilical region) is carried out and then the colon is removed. Next is the fashioning of the ileal pouch ( $\mathrm{j}$-pouch) outside of the body using the GIA-60 and the "head" of the Premium CEA 31 is positioned in the declive part of the j-pouch. The hemostasis is checked and, after having repositioned the ileal pouch on the inside of the abdominal cavity one proceeds with the closure of the minilaparotomy. We conclude with the fashioning of the ileo (pouch)-rectal anastomosis (with the "Premium CEA 31) and the successive check of intra-operative hold (an insufflation test via the introduction of saline solution into the pelvis and simultaneous insufflation with an endoscope inserted in the rectum). A drainage tube is positioned using the site of a trocar (generally the one positioned on the patient's left-hand-side on the transverse umbilical line) which is positioned in proximity to the anastomosis. The surgery ends with the removal of the trocars under direct vision.

\section{Results}

There were no post-operative complications. The patient resumed the normal diet on the 5th post-operative day after the carrying out of an X-ray of the pouch with Gastrografin through the introduction of a trans-anal Foley, that did not show extra-luminal leakage of the radiocontrast agents, and she was discharged on the 8th post-operative day. The patient underwent a routine clinical check-up at one month and at three months following the surgery: she referred a good satisfaction. After the third month the patient reported excellent results with $2-3$ evacuations a day and soft but formed stool with no mention of abdominal pain, nausea or flatulence and, therefore, an overall improvement in the quality of life.

\section{Discussion}

Chronic slow transit constipation (STC) represents a syndrome characterised by constipation refractory to pharmacologic treatment, accompanied by chronic abdominal pain, nausea and flatulence, which above all affects young female patients [4,5]. The physiopathologic mechanisms that determine this condition are still not completely clear, although it has been hypothesised that a dysfunction of the mioenteric plexus accompanied by altered levels of various neuroendocrine peptides, such as pancreatic polypeptide, peptide YY, neuropeptide Y, substance $\mathrm{P}$, neurotensin, gastrin-releasing peptide, enkephalin, somatostatin and vasoactive intestinal polypeptide [68].

Only a minority of patients affected by this syndrome require surgical treatment and, for this reason, studies are still limited. Despite this, published material is in accordance with a preference to treatment using TC with IRA and SC with CRA. Effectively, both these types of procedure have revealed optimal results in terms of morbidity, mortality and quality of life, with a success rate of approximately $90 \%[9,10]$. In particular, the TC with IRA procedure is the most commonly used, even though SC with CRA, saving the end ileo, the cecorectal valve and the cecum, seem to guarantee a better absorption of electrolytes, bile salts, B12 vitamin and water [11-13]. The number of bowel movements after the third month following surgery, is around 2 - 3 times a day for both cases. The choice of a laparoscopic total colectomy with ileal j-pouch comes from the desire to further reduce the number of daily evacuations, due to the creation of a reservoir able to contain greater faecal volume in respect to the direct ileorectal anastomosis and cecorectal anastomosis. Although in this our experience we have reported good results, it is also true that a case report is not sufficient in drawing conclusions in merit of superiority or 
inferiority of one treatment with respect to another, therefore further studies are needed to be able to establish which procedure of laparoscopic surgery is to be preferred for the treatment of chronic constipation refractory to medical therapy.

\section{REFERENCES}

[1] M. F. Piccirillo, P. Reisman and S. D. Wexner, "Colectomy as Treatment for Constipation in Selecte Patients," British Journal of Surgery, Vol. 82, No. 7, 1995, pp. 899901.

[2] M. El-Shaly, "Chronic Idiopatic Slow Transit Constipation: Pathophysiology and Management," Colorectal Disease, Vol. 5, No. 4, 2003, pp. 288-296. doi:10.1046/j.1463-1318.2003.00498.x

[3] G. Conzo, A. Allaria, F. Stanzione, G. Rossetti, G. Candela, C. Mauriello, L. Fei and L. Santini, "Laparoscopic Treatment of Chronic Slow Transit Constipation. Report of Three Cases and Review of Literature,” Annali Italiani di Chirurgia, Vol. 83, No. 2, 2012, pp. 113-117.

[4] M. Candelli, E. C. Nista, M. A. Zocco and A. Gasbarrini, "Idiopatic Chronic Constipation. Pathophysiology, Diagnosis and Treatment,” Hepato-Gastroenterology, Vol. 48, No. 40, 2001, pp. 1050-1057.

[5] A. Wald, "Constipation,” Medical Clinics of North America, Vol. 84, No. 4, 2000, pp. 1231-1246. doi:10.1016/S0025-7125(05)70284-4

[6] C. H. Knowles, S. M. Scott and P. J. Lunniss, "Slow Transit Constipation: A Disorder of Pelvic Autonomic Nerve?” Digestive Diseases and Sciences, Vol. 46, No. 3, 2001, pp. 389-401. doi:10.1023/A:1005665218647

[7] W. R. Schouthen, F. J. W. ten Kate, E. J. R. de Graaf, E. C. A. M. Gilberts, J. L. Simons and P. Kluck, "Visceral Neuropathy in Sloe Transit Constipation: An Immuno- histochemical Investigation with Monoclonal Antibodies against Neurofilament," Diseases of the Colon \& Rectum, Vol. 36, No. 12, 1993, pp. 1112-117. doi:10.1007/BF02052258

[8] M. El-Salhy and O. Norrgard, "Colonic Neuroendocrine peptide Levels in Patients with Chronic Idiopathic Slow Transit Constipation," Upsala Journal of Medical Sciences, Vol. 103 No. 3, 1998, pp. 223-230. doi:10.3109/03009739809178951

[9] D. C. Nyam, J. H. Pemberton, D. M. Ilstrup and D. M. Rath, "Long Term Results of Surgery for Chronic Constipation,” Diseases of the Colon \& Rectum, Vol. 40, No. 3, 1997, pp. 273-79. doi:10.1007/BF02050415

[10] R. M. Mollen, H. C. Kuijpers and T. Claassen, "Colectomy for Slow-Transit Constipation: Preoperative Functional Evaluation Is Important But Not a Guarantee for a Successful Outcome,” Diseases of the Colon \& Rectum, Vol. 44 No. 4, 2001, pp. 577-80. doi:10.1007/BF02234332

[11] F. Marchesi, L. Sarli, L. Percalli, G. E. Sansebastiano, L. Veronesi, D. Di Mauro, C. Porrini, M. Ferro and L. Roncoroni, "Subtotal Colectomy with Antiperistaltic Cecorectal Anastomosis in the Treatment of Slow-Transit Constipation: Long-Term Impact on Quality of Life,” World Journal of Surgery, Vol. 31, No. 8, 2007, pp. 1658-1664. doi:10.1007/s00268-007-9111-6

[12] C. Q. Jiang, Q. Qian, Z. S. Liu, G. Bangoura, K. Y. Zheng and Y. H. Wu, "Subtotal Colectomy with Antiperistaltic Cecoproctostomy for Selected Patients with Slow Transit Constipation-From Chinese Report," International Journal of Colorectal Disease, Vol. 23, No. 12, 2008, pp. 1251-1256. doi:10.1007/s00384-008-0552-7

[13] R. Levitan, S. J. Fordtran, B. A. Burrows and F. J. Ingelfinger, "Water and Sale Absorbation in Humane Colon," Journal of Clinical Investigation, Vol. 41, No. 9, 1962, pp. 273-279. 